\title{
An inerter-based active vibration isolation system
}

\author{
Neven Alujević ${ }^{1}$, Damjan C̆akmak $^{1, *}$, Hinko Wolf ${ }^{1}$, and Marko Jokić ${ }^{1}$ \\ ${ }^{1}$ Fakultet strojarstva i brodogradnje, Sveučilište u Zagrebu, Ivana Lučića 5, 10000 Zagreb, Croatia
}

\begin{abstract}
This paper presents a theoretical study on passive and active vibration isolation schemes using inerter elements in a two degree of freedom (DOF) mechanical system. The aim of the work is to discuss basic capabilities and limitations of the vibration control systems at hand using simple and physically transparent models. Broad frequency band dynamic excitation of the source DOF is assumed. The purpose of the isolator system is to prevent vibration transmission to the receiving DOF. The frequency averaged kinetic energy of the receiving mass is used as the metric for vibration isolation quality. It is shown that the use of inerter in the passive and active vibration isolation schemes considered enhances the isolation effect.
\end{abstract}

\section{Introduction}

Inerter is a one port element in mechanical networks which resists relative acceleration across its two terminals $[1,2]$. The coefficient of this resistance is called inertance and is measured in kilograms. An appealing property of inerters is that they can be designed and realized in practice having their inertance significantly larger than their mass $[1,2]$. This opens many interesting possibilities so that many authors reported on how to design and use inerters to suppress mechanical vibrations [1-20].

The concept of "relative mass" has been considered by Schönfeld [21] in connection with mechanicalelectrical analogies. He mentioned the possibility of a two-terminal mechanical inertance and gave a rudimentary scheme of a physical realization of the concept. Smith [1], and Smith and Wang [2] developed this idea by investigating how to design such a device in practice and pointed out a number of peculiarities that the new element brings into a mechanical network. The authors instilled that inerter is the analogue of the capacitor element in electrical networks [2]. Therefore, adding the inerter to classical dampers and springs fills an empty niche enabling a complete synthesis of passive mechanical networks $[2,21]$.

Smith and Wang designed their inerter using a plunger sliding in a cylinder which drives a flywheel through a rack, pinion and gears [2]. Alternatively, an electromagnetic transducer (voice coil, linear motor) can be shunted with electrical impedance. If the total shunt impedance is properly tuned, then the whole electromechanical network theoretically behaves exactly as if it incorporated an ideal inerter mounted in series with a parallel spring damper-pair [3]. In this context, self-powered configurations employing a simultaneous active control and energy harvesting have been considered to synthesize mechatronic inerters [3]. Another type of mechatronic inerter utilizes a rotary DC motor shunted with an appropriate electrical circuit [4]. An inertance-like behaviour can also be accomplished through a scheme in which hydraulic fluid is accelerated $[5,6]$ with a piston which pushes the fluid through a helical channel [6].

Inerters can be very useful in vibration absorber systems. Performance of vibration absorbers, especially Tuned Mass Dampers (TMDs) is known to very much depend on the proof mass added to a primary structure to reduce its vibration. As this mass is added to structures exclusively to control their vibrations, it is penalized in lightweight automotive and aerospace applications [13, 14]. In this context the use of inerter elements can be interesting given the fact that their inertance can be significantly larger than their mass. Consequently a number of new concepts have arisen. These include tuned inerter damper (TID), tuned mass-damper-inerter (TMDI), and inerter-based dynamic vibration absorber (IDVA) [15-19]. Various applications have been considered using tuned inerter dampers including vibration reduction of cables in cable-stayed bridges $[15$, $16]$.

Dynamic vibration absorbers can be made active by using inertial actuators with a velocity or velocity + displacement feedback control scheme. Inertial actuators are typically designed with a low mounted natural frequency in order to widen the range of frequencies where they can efficiently actuate. However, the low natural frequency is usually associated with increased static sags. This limits the applicability of inertial actuators in presence of large accelerations (i.e. vehicle manoeuvring or centrifugal accelerations in rotating structures [22-24]).

Zilletti investigated an active vibration absorber system in which the inerter is attached in parallel with the suspension spring, damper, and the actuator [20]. The author has shown that with such a design it is possible to reduce the natural frequency of the actuator without increasing the proof mass or reducing the

\footnotetext{
* Corresponding author: damjan.cakmak@,fsb.hr
} 
suspension stiffness. He considered only an idealized inerter element, which neglects the inertial, stiffness and damping of the gearing mechanism. However, Kras and Gardonio studied the effective weight and dynamic effects of an inerter element composed by a single flywheel which is either pinned or hinged to the base mass or to the proof mass of the actuator [25].

Inerters can also be very useful in vibration isolation systems. In this sense, many authors focused their efforts on improving vehicle suspension systems using inerters [2], [7-10]. Further applications of inerters include vibration isolation in civil engineering structures, such as multi-storey buildings under earthquake base excitation [11]. In vibration isolation problems it is often necessary to tune the impedance of the isolator elements based on some optimization criteria. This can be done by either minimizing maxima of the response (minimax or $\mathrm{H}_{\infty}$ optimization), or by minimizing the energy in the response signals $\left(\mathrm{H}_{2}\right.$ optimization) [12].

In this paper an active vibration isolation problem is considered. It is shown that the use of inerter can significantly improve the stability and performance of the active vibration isolation system in certain situations. In particular, it is shown analytically on a simplified model problem that the use of inerter enables successful active vibration isolation in a family of mechanical systems that are otherwise difficult to control. This family of systems has been referred to as subcritical 2 DOF systems [26]. Subcritical systems are those characterized by the natural frequency of the receiving body larger than that of the source body. In such vibration isolation problems the use of inerter is shown to stabilize the feedback loop and therefore to enable a remarkable active vibration isolation effect. In addition to the active vibration isolation system, an inerter-based and an inerter-free passive isolator scheme are proposed and analysed, with the aim of establishing fair benchmarks for the evaluation of the performance of the active isolators studied later in the paper.

In each system, either active of passive, tuneable parameters are adjusted in order to minimize the kinetic energy of the receiving body per unit, spectrally white, dynamic excitation of the source body.

\section{Mathematical model}

As shown in Fig. 1., the problem studied is represented by a lumped parameter two degree of freedom (DOF) mechanical system. The system consists of two masses $m_{1}$ and $m_{2}$ coupled by a spring $k_{2}$, a viscous damper $c_{2}$ and an inerter of inertance $b_{2}$. The inerter produces a force proportional to the relative acceleration between masses $m_{1}$ and $m_{2}$. The two masses are attached to fixed reference bases via the two mounting springs $k_{1}$ and $k_{3}$. The lower mass $m_{1}$ is excited by the disturbance force $F_{1}$. It is assumed that the force $F_{1}$ has characteristics of an ideal white noise and that the power spectral density (PSD) of the force equals one over all frequencies. The effects of dampers between the source mass $m_{1}$ and the ground and between the receiving mass $m_{2}$ and the ground are neglected i.e. $c_{1} \approx c_{3} \approx 0$.

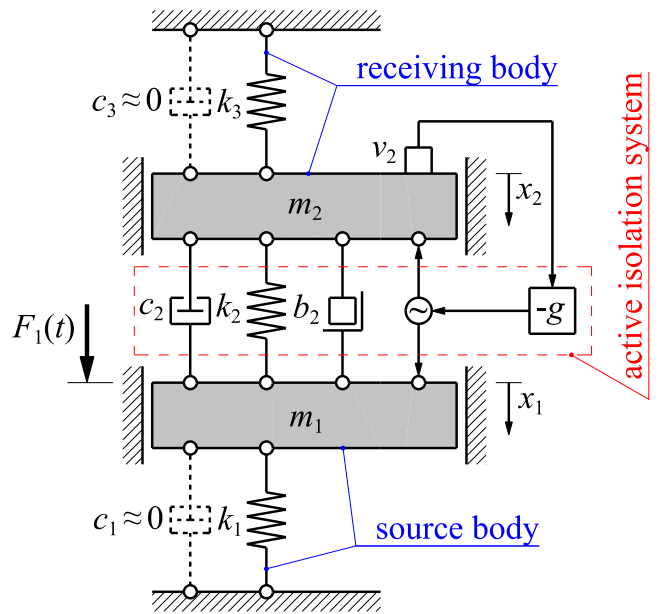

Fig. 1. The two degree of freedom lumped mass active vibration isolation system.

More complex systems may also be representable by the general configuration shown in Fig. 1. [27-30].

The active part of the vibration isolation system is realized through a skyhook damping unit $[30,31]$. The velocity sensor is mounted onto mass $m_{2}$ in order to realize a disturbance rejection control scheme. In this scheme the actuator is driven with a signal proportional to the negative absolute velocity of the receiving body amplified by a constant control gain $g$. The equations of motion can be written in the matrix form as [32]

$$
\mathbf{M} \ddot{\mathbf{x}}+\mathbf{C} \dot{\mathbf{x}}+\mathbf{K x}=\mathbf{F},
$$

where $\mathbf{M}$ is the mass matrix, $\mathbf{K}$ is the stiffness matrix, $\mathbf{C}$ is the damping matrix, $\mathbf{x}(t), \dot{\mathbf{x}}(t)$ and $\ddot{\mathbf{x}}(t)$ are the displacement, velocity and acceleration column vectors respectively, and $\mathbf{F}(t)$ is excitation column vector. Assuming a simple harmonic excitation and expressing the excitation and the steady-state response in the exponential form $\mathbf{F}(t)=\hat{\mathbf{F}} \mathrm{e}^{\mathrm{j} \omega t}$ and $\mathbf{x}=\hat{\mathbf{x}} \mathrm{e}^{\mathrm{j} \omega t}$, Eq. (1) can be differentiated with respect to time $t$ and written as

$$
\dot{\mathbf{x}}(\mathrm{j} \omega)=\mathbf{Y}(\mathrm{j} \omega) \mathbf{F}(\mathrm{j} \omega),
$$

where $\dot{\mathbf{x}}(\mathrm{j} \omega)=\mathrm{j} \omega \mathbf{x}(\mathrm{j} \omega)$ is the velocity vector, $\mathbf{S}(\mathrm{j} \omega)$ is the dynamic stiffness matrix and $\mathbf{Y}(\mathrm{j} \omega)=\mathrm{j} \omega \mathbf{S}^{-1}(\mathrm{j} \omega)$ is the mobility matrix containing four frequency response functions (FRFs) between velocities and forces. With the aim of more general approach, the element, $Y_{21}$, of the mobility matrix in Eq. (2) can be expressed in the following dimensionless form

$$
r_{21}(\mathrm{j} \Omega)=\frac{B_{0}+(\mathrm{j} \Omega) B_{1}+(\mathrm{j} \Omega)^{2} B_{2}+(\mathrm{j} \Omega)^{3} B_{3}}{A_{0}+(\mathrm{j} \Omega) A_{1}+(\mathrm{j} \Omega)^{2} A_{2}+(\mathrm{j} \Omega)^{3} A_{3}+(\mathrm{j} \Omega)^{4} A_{4}},
$$

where coefficients $A_{0} \ldots A_{4}$ and $B_{0} \ldots B_{3}$ are given by

$$
\begin{array}{ll}
A_{0}=\mu_{1}\left(\alpha \beta \mu_{1}+\alpha+\beta\right) & B_{0}=0 \\
A_{1}=2 \eta_{2}\left(\beta \mu_{1}+\lambda+1\right) & B_{1}=\alpha \mu_{1}, \\
A_{2}=\mu_{1}\left(\alpha \mu_{1}+\beta \mu_{1} \mu_{2}+\alpha+\beta+\mu_{2}+1\right) & B_{2}=2 \eta_{2} \\
A_{3}=2 \eta_{2}\left(\mu_{1}+\lambda+1\right) & B_{3}=\mu_{1} \mu_{2} \\
A_{4}=\mu_{1}\left(\mu_{1} \mu_{2}+\mu_{2}+1\right) &
\end{array}
$$$$
B_{1}=\alpha \mu_{1},(4 \mathrm{a}-\mathrm{i})
$$

where

$$
\alpha=\left(\frac{\Omega_{2}}{\Omega_{1}}\right)^{2}, \beta=\left(\frac{\Omega_{3}}{\Omega_{1}}\right)^{2}, \eta_{2}=\frac{c_{2}}{2 \sqrt{m_{1} k_{1}}}, \lambda=\frac{g}{c_{2}}, \mu_{1}=\frac{m_{2}}{m_{1}}, \mu_{2}=\frac{b_{2}}{m_{2}}, \Omega=\frac{\omega}{\Omega_{1}},
$$
and $r_{21}=m_{1} \Omega_{1} Y_{21}$ is now the dimensionless transfer mobility. In Eqs. (5a-g), $\alpha$ and $\beta$ are squared natural frequency ratios, $\eta_{2}$ is the damping ratio, $\lambda$ is the 
feedback gain normalized with respect to the passive damping coefficient, and $\mu_{1}$ and $\mu_{2}$ are the mass and inertance ratios respectively. Furthermore, $\Omega$ is dimensionless circular frequency normalized with respect to the natural frequency of the uncoupled source body $\Omega_{1}$ (as if the source body was uncoupled by removing spring $k_{2}$ ), $\Omega_{3}$ is the natural frequency of the uncoupled receiving body (as if the receiving body was uncoupled by removing spring $k_{2}$ ), and $\Omega_{2}$ is the natural frequency of the receiving body as if it was attached to a fixed reference base through the spring of stiffness $k_{2}$ only. The three natural frequencies $\Omega_{1} \ldots \Omega_{3}$ are thus

$$
\Omega_{1}=\sqrt{\frac{k_{1}}{m_{1}}}, \Omega_{2}=\sqrt{\frac{k_{2}}{m_{2}}}, \Omega_{3}=\sqrt{\frac{k_{3}}{m_{2}}} .
$$

Given that the excitation force $F_{1}$ with unit power spectral density (PSD) has been assumed, the specific kinetic energy of the receiving body (per unit mass, per unit excitation force) can now be calculated as

$$
I_{k}=\int_{-\infty}^{\infty}\left|r_{21}(\mathrm{j} \Omega)\right|^{2} \mathrm{~d} \Omega
$$

according to the Parseval's identity. The specific kinetic energy index $I_{k}$ is used throughout this study as the measure of the performance of broad frequency band vibration isolation. Formulae for integrating ratios of polynomial functions of a complex variable can be found in [33]. Throughout the paper it is investigated how can the kinetic energy of the receiving body be minimised by minimising the specific kinetic energy index in Eq. (7).

\section{Passive control}

In this section, two benchmark passive vibration isolation schemes are considered. In the first scheme, an inerter is not employed and in the second, an inerter of dimensionless inertance $\mu_{2}$ is considered.

\subsection{Without inerter}

The modulus of the transfer mobility $\left|r_{21}\right|$ is shown in Fig. 2. for three values of the passive damping ratio $\eta_{2}$ : a relatively small one (solid line), a medium (dashed line) and a large damping ratio (dash-dotted line).

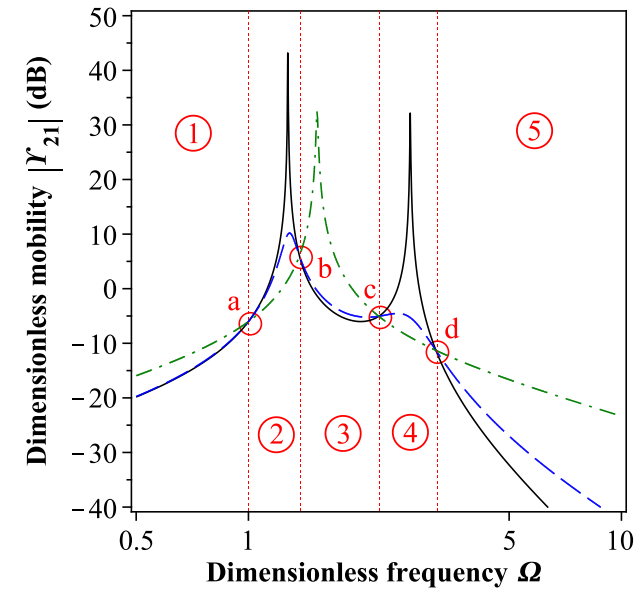

Fig. 2. Isolation system performance without inerter $b_{2}$ $\left(\mu_{2}=0\right)$ : Transfer mobility function $\left|r_{21}(\mathrm{j} \Omega)\right|, \eta_{2}=\eta_{2 \mathrm{opt} 1} / 100$

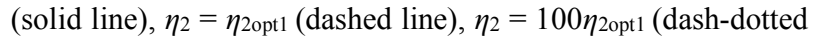
line).
Parameters that characterize this example system from Fig. 1. are $\alpha=2, \beta=5$ and $\mu_{1}=1 / 2$. The variation of the passive damping ratio shows that it is possible to optimise the system so that the receiving body vibration measured through the metrics defined by Eq. (7) is minimised. This is because: i) for a small damping ratio the response is very large at resonances (solid line); ii) for a very large damping ratio the damper locks the source and receiving bodies and a new lightly damped resonance is generated (dash-dotted line). It is therefore best to use: iii) the optimal damping ratio which minimises the kinetic energy of the receiving body. The optimal damping ratio corresponds to the dashed line in Fig. 2. Also shown in the figure are five frequency ranges, $1-5$, in which the variation of the damping ratio causes either an increase or a reduction of the amplitude of the transfer mobility function. The corresponding bordering frequencies are designated by letters a-d. In particular, around resonances the increase in damping decreases the vibration transmission, but elsewhere it increases the vibration transmission. Thus the optimal damping ratio exists.

\subsection{With inerter}

Fig. 3. shows the amplitude of the dimensionless transfer mobility $\left|r_{21}\right|$ for the same parameters as in Fig. 2., except that an inerter is now employed. A minimisation of the specific kinetic energy index has been carried out with respect to the inertance and the damping. The minimisation indicates that there is an optimal inertancedamping pair which minimises the receiving body kinetic energy. The results in Fig. 3 are shown for the optimised inertance, however the damping ratio has been varied from suboptimal towards the optimal one and above it. Anti-resonance effect at the dimensionless frequency of $\Omega_{\mathrm{A}}=\sqrt{\alpha / \mu_{2}}$ (corresponding to the dimensional frequency $\omega_{\mathrm{A}}=\sqrt{k_{2} / b_{2}}$ ) is observed (solid line) which is a result of using the inerter.

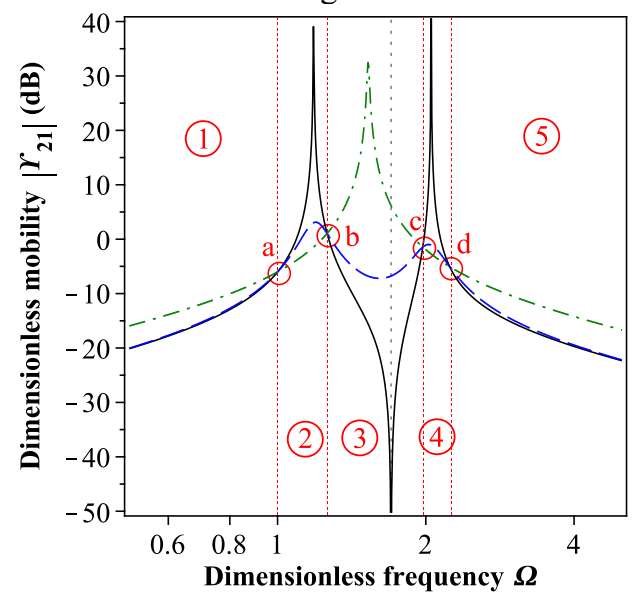

Fig. 3. Isolation system performance with inerter $b_{2}\left(\mu_{2}=\mu_{2 \mathrm{opt}}\right)$ : Transfer mobility function $\left|r_{21}(\mathrm{j} \Omega)\right|, \eta_{2}=0$ (solid line), $\eta_{2}=\eta_{2 \mathrm{opt} 2}\left(\right.$ dashed line), $\eta_{2}=100 \eta_{2 \mathrm{opt} 2}($ dash-dotted line).

The use of inerter improves the broadband passive vibration isolation performance by $\sim 3 \mathrm{~dB}$ for the case considered where $\eta_{2}=\eta_{2 \text { opt2 }}$ and $\mu_{2}=\mu_{2 \text { opt }}$ in comparison to the system studied in the previous subsection. This 
improvement certainly depends on the parameters that characterise the system without inerter and the particular vibration isolation problem at hand.

\section{Active control}

\subsection{Stability analysis}

In this section, two active vibration isolation schemes are analysed with respect to the stability of the feedback control. In the first scheme the inerter is not used, and in the second it is. The stability study is carried out using the Routh-Hurwitz criterion [34, 35].

If the inerter is not used then the analysis of the stability indicates that there are two basic families of systems. The first family can be referred to as supercritical and it is characterized by $\beta<1$ (the natural frequency of the source body is higher than that of the receiving body). The systems belonging to this group allow for the implementation of unconditionally stable active vibration isolation scheme based on the direct feedback of the absolute velocity of the receiving body even if the inerter is not used. The second family is characterized by $\beta>1$ and it can be referred to as subcritical. The systems belonging to this group do not allow for the implementation of unconditionally stable absolute velocity feedback scheme, as the feedback loop is only conditionally stable with a limited maximum feedback gain $g$, i.e. $\lambda$.

Fig. 4. shows the Hurwitz principal diagonal minors plotted as a function of the dimensionless feedback gain $\lambda$ when no inerter is used. The system is subcritical with characteristic parameters $\alpha=2, \mu_{1}=1 / 2, \eta_{2}=1$ and $\beta=$ 5 .

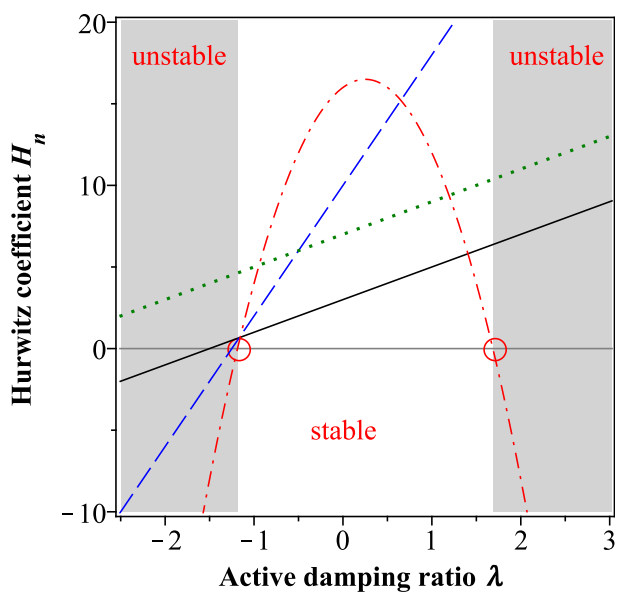

Fig. 4. Dependence of Hurwitz coefficients $H_{1}$ (solid line), $H_{2}$ (dashed line), $H_{3}$ (dash-dotted line) and $A_{1}$ (dotted line) magnitude on active damping ratio $\lambda$ without inerter $b_{2}\left(\mu_{2}=0\right)$ : $\beta_{\mathrm{II}}>1$.

A limited stability range as a function of $\lambda$ is observed. By a detailed analysis of the sign of the Hurwitz principal diagonal minors it can be shown that by including the inerter with inertance $\mu_{2}>\alpha$, a subcritical system becomes unconditionally stable for any chosen positive $\lambda$. This is illustrated in Fig. 5. on the same passive system but this time employing an inerter with dimensionless inertance of $\mu_{2}=4$.

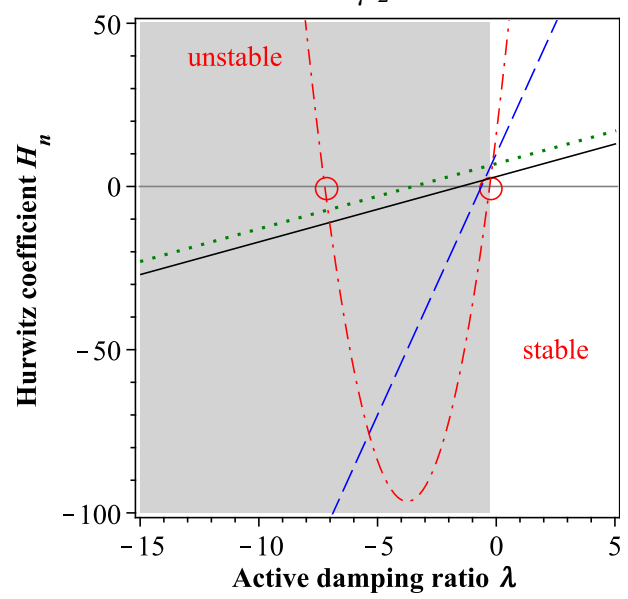

Fig. 5. Dependence of Hurwitz coefficients $H_{1}$ (solid line), $H_{2}$ (dashed line), $H_{3}$ (dash-dotted line) and $A_{1}$ (dotted line) magnitude on active damping ratio $\lambda$ with inerter $b_{2}\left(\mu_{2} \neq 0\right)$ and $\beta_{\mathrm{II}}>1: \mu_{2}>\alpha$.

\subsection{Performance without inerter}

Fig. 6. shows the amplitude of the dimensionless transfer mobility $\left|r_{21}\right|$ of a subcritical system characterised by the parameters $\alpha=1 / 2, \beta=5$ and $\mu_{1}=1 / 2$ and $\eta_{2}=0.02$. The dimensionless feedback gain $\lambda$ is varied from zero to 1.5 .

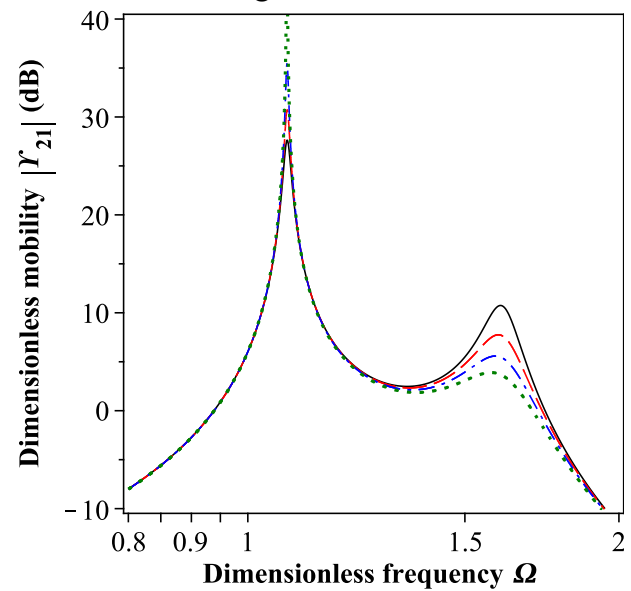

Fig. 6. Subcritical isolation system performance without inerter $b_{2}\left(\mu_{2}=0\right)$ and $\beta_{\mathrm{II}}>1$ : Transfer mobility function $\left|r_{21}(\mathrm{j} \Omega)\right|, \lambda=0$ (solid line), $\lambda=0.5$ (dashed line), $\lambda=1$ (dash-dotted line), $\lambda=1.5$ (dotted line).

A significant overshoot can be observed in the vicinity of the first dimensionless natural frequency $\Omega_{n 1}$ for rising $\lambda$, which also results with system instability if the feedback gain is further increased. Thus the performance of the active control scheme considered is jeopardised by the limited stability of the feedback loop.

\subsection{Performance with inerter}

Fig. 7. shows the subcritical system transfer mobility amplitude $\left|\gamma_{21}\right|$ with $\alpha=1 / 2, \beta=2, \mu_{1}=1 / 2$ and $\eta_{2}=0.02$, equipped with an inerter of inertance $\mu_{2}=2$. Therefore, an inertance large enough to stabilize the feedback loop is used $\left(\mu_{2}>\alpha\right)$. 


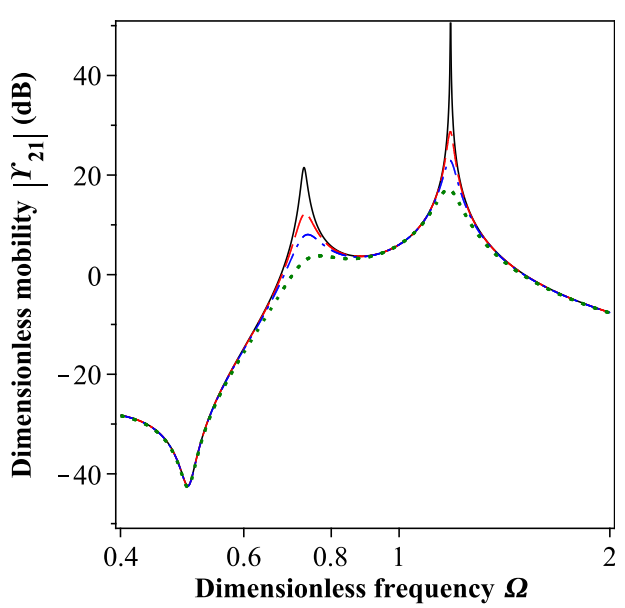

Fig. 7. Subcritical isolation system performance with inerter $b_{2}$ $\left(\mu_{2} \neq 0\right), \beta_{\mathrm{II}}>1$ and $\mu_{2}>\alpha$ : Transfer mobility function $\mid r_{21}(\mathrm{j} \Omega)$, $\lambda=0$ (solid line), $\lambda=5$ (dashed line), $\lambda=10$ (dash-dotted line), $\lambda=20$ (dotted line).

It can be seen that with an increase in the dimensionless gain $\lambda$, the amplitude of the dimensionless mobility $\left|r_{21}\right|$ decreases at all frequencies, which indicates that the desired vibration isolation effect is now accomplished. Anti-resonance is observed below the first natural frequency which is due to the use of the inerter in the isolator. The amplitude at this frequency does not increase with the increase in the dimensionless gain.

It should be noted that adding the inerter into the isolator effectively generates a sort of relative acceleration feedback that stabilizes the control loop. It is important to mention that direct acceleration feedback would probably not be possible in practice due to very pronounced stability problems [36]; therefore the passive element which mimics such feedback is very useful.

\section{Conclusions}

In this paper, an inerter-based active vibration isolation system is presented. Two fundamental passive benchmark isolators are also investigated, one not employing the inerter and the other employing the inerter. The methodology is studied on a simple two degree of freedom system so that many conclusions can be drawn based on analytically derived expressions. Such a simplified system can be seen as a reduced order model of a potentially more complex structure. It is shown in the paper that the vibration isolation performance of the fundamental passive isolator not employing the inerter can be improved by adding the inerter in parallel with the isolator spring and damper. By investigating the stability of the active control when no inerter is used, it is found that there are two fundamental families of vibration isolation problems. With the first family (supercritical systems), which is characterized by the natural frequency of the uncoupled source body larger than the natural frequency of the uncoupled receiving body, large feedback gains can be used without compromising the stability of the feedback control system. This results in a convincing broadband vibration isolation effect. With the second family of systems (subcritical systems), the natural frequency of the uncoupled source body is below the natural frequency of the uncoupled receiving body. The range of stable feedback gains is limited which results in poor vibration isolation performance. However with the inclusion of the inerter, broadband active vibration isolation can also be achieved in the subcritical family of systems. In fact, it is shown that the minimum inertance to stabilize the loop is proportional to the stiffness of the isolator spring and inversely proportional to the squared natural frequency of the source body.

This project has received funding from the European Union's Horizon 2020 research and innovation programme under the Marie Sklodowska-Curie grant agreement no. 657539 STARMAS.

\section{References}

1. M.C. Smith, IEEE T. Autom. Control 47, 1648-1662 (2002)

2. M.C. Smith, F.C. Wang, Veh. Syst. Dyn. 42, 235257 (2004)

3. A. Gonzalez-Buelga, L.R. Clare, S.A. Neild, J.Z. Jiang, D.J. Inman, Smart Mater. Struct. 24, 1-10 (2015)

4. D. Li, Y.-Z. Liu, App. Mech. Mater. 620, 28-32 (2014)

5. F.-C. Wang, M.-F. Hong, T.-C. Lin, Proceedings of the Institution of Mechanical Engineers, Part C: J. Mech, Eng. Sci. 225, 66-72 (2011)

6. S.J. Swift, M.C. Smith, A.R. Glover, C. Papageorgiou, B. Gartner, N.E. Houghton, Int. J. Control, Systems, Modelling and Feedback Control: A Special Issue Dedicated to Sir Alistair G.J. MacFarlane 86, 2035-2051 (2013)

7. M.Z.Q. Chen, Y. Hu, C. Li, G. Chen, IEEE T. Contr. Syst. T. 23, 1571-1577 (2015)

8. M.Z.Q. Chen, K. Wang, Y. Zou, J. Lam, IEEE T. Autom. Control 58, 1841-1846 (2013)

9. M.Z.Q. Chen, Y. Hu, C. Li, G. Chen, J. Vib. Acoust. 138, 1-11 (2016)

10. A. Kuznetsov, M. Mammadov, I. Sultan, E. Hajilarov, Arch. Appl. Mech. 81, 1427-1437 (2011)

11. I.F. Lazar, S.A. Neild, D.J. Wagg, Earthq. Eng. Struct. D. 43, 1129-1147 (2014)

12. Y. Hu, M.Z.Q. Chen, Z. Shu, L. Huang, J. Sound Vib. 346, 17-36 (2015)

13. T. Bein, D. Mayer, J. Bös, INTER-NOISE 2012 5, 3759-3771 (2012)

14. T. Bein, J. Bös, S. Herold, D. Mayer, T. Melz, M. Thomaier, Aerosp. Sci. Technol. 12, 62-73 (2008)

15. I.F. Lazar, S.A. Neild, D.J. Wagg, Conference Proceedings of the Society for Experimental Mechanics Ser. 2, 433-441 (2015)

16. I.F. Lazar, S.A. Neild, D.J. Wagg, Eng. Struct. 122, 62-71 (2016) 
17. P. Brzeski, T. Kapitaniak, P. Perlikowski, J. Sound Vib. 349, 56-66 (2015)

18. P. Brzeski, E. Pavlovskaia, T. Kapitaniak, P. Perlikowski, Int. J. Non-Lin. Mech. 70, 20-29 (2015)

19. Y. Hu, M.Z.Q. Chen, Int. J. Mech. Sci. 99, 297-307 (2015)

20. M. Zilletti, J. Sound Vib. 369, 16-28 (2016)

21. J.C. Schönfeld, Appl. Sci. Res. 3, 417-450 (1954)

22. G. Zhao, N. Alujević, B. Depraetere, G. Pinte, J. Swevers, P. Sas, J. Sound Vib. 348, 15-30 (2015)

23. G. Zhao, N. Alujević, B. Depraetere, G. Pinte, P. Sas, J. Intel. Mat. Syst. Str. 27, 1584-1599 (2016)

24. G. Zhao, N. Alujević, B. Depraetere, P. Sas, J. Intel. Mat. Syst. Str. 26, 1995-2010 (2015)

25. A. Kras, P. Gardonio, J. Sound Vib. 402, 31-50 (2017)

26. N. Alujević, H. Wolf, P. Gardonio, I. Tomac, J. Sound Vib. 330(21), 4981-4997 (2011)

27. R.M. Chalasani, ASME Symposium on Simulation and Control of Ground Vehicles and Transportation Systems, AMD-vol. 80, DSC-vol. 2, 187-204 (1986)

28. A. Caiazzo, N. Alujević, B. Pluymers, W. Desmet, J. Phys. Conference series 744, 012026 (2016).

29. A. Preumont, Vibration Control of Active Structures, 2nd Edition (Kluwer Academic, London, 2002, Chapter 6, "Active vibration isolation")

30. C.E. Kaplow, J.R. Velman, AIAA J. Guid. Control Dynam. 3(3), 227-233 (1980)

31. D.C. Karnopp, A.K. Trikha, Trans. ASME, J. Eng. Ind., Ser. B 91, 1128-1132 (1969)

32. W.T. Thomson, Theory of vibration with applications (Prentice Hall, London, 1997)

33. D.E. Newland, An introduction to Random Vibrations, Spectral \& Wavelet Analysis, Third Edition (Dover Publications Inc, New York, 2005)

34. E.J. Routh, A Treatise on the Stability of a Given State of Motion: Particularly Steady Motion (Macmillan and Co., London, 1877)

35. A. Hurwitz, Math. Ann. 46, 273-284 (1895)

36. N. Alujević, I. Tomac, P. Gardonio, J. Sound Vib. 331, 2731-2728 (2012) 